\title{
Orofacial trauma management in a wind instrument player
}

\author{
Miguel Pais Clemente ${ }^{*}$, Joaquim Gabriel Mendes ${ }^{2}$, André Moreira ${ }^{3}$, Afonso Pinhão Ferreira ${ }^{4}$ and José Manuel Amarante \\ ${ }^{1}$ Dentist, PhD Student, Faculty of Dental Medicine Porto, Portugal \\ ${ }^{2}$ INEGI, Labiomep, Faculty of Engineering, University of Porto, Portugal \\ ${ }^{3}$ Dentist, Specialization Student in Prosthodontics, Faculty of Dental Medicine Porto, Portugal \\ ${ }^{4}$ Department of Orthodontics, Faculty of Dental Medicine, University of Porto, Portugal \\ ${ }^{5}$ Department of Surgery, Faculty of Medicine, University of Porto, Portugal
}

\begin{abstract}
Background: Regarding the orofacial structures, musical performance in wind instrumentalists can promote undesirable pressures on teeth which can be the origin of orofacial pain.

Objective: This article addresses the therapeutic options for a clarinet player, which has tooth mobility and orofacial pain due to the occurrence of an orofacial trauma

Methods: The clarinetist had an alteration in his embouchure due to an orofacial trauma and consequent difficulty on playing his instrument. The authors had to deal with the mobility and restoration of the compromised endodontically tooth. After the stages of the oral rehabilitation a homemade fitted appliance, the Embouchure Stabilization Mouthpiece Appliance - ESMA was made to reduce abnormal and undesired forces on the upper central incisors, evaluated by piezoresistive sensors.

Results: The implemented treatment of the central incisor is crucial for the embouchure and musical performance of the clarinetist. The dental treatment fulfilled the necessary conditions for its maintenance; otherwise, the placement of a removable prosthodontics on the upper right central incisor would probably not allow the clarinetist to play anymore. However, the proposed device, ESMA, was constructed for the anterior upper incisal block, allowed the musician to perform and continuing playing the clarinet.
\end{abstract}

Conclusions: The evolution of dental techniques will enhance better solutions for patients in general. There will be always uncertainty towards the response of the biological tissues; however, by understanding the forces that are being applied during musical performance, one may contribute to player's well-being.

\section{Introduction}

During musical performance, wind instrumentalists have to play different repertoires, which obliges to a high demand of neurologic and physical activity. Only a good synchronization of the mind and the body will allow the musician to play the correct notes in the precise time.

It is of common knowledge that musicians are subjected to high physical demand during training and performance that may eventually lead to back, cervical, orofacial pain in addition to neurologic disorders like focal dystonia, carpal tunnel syndromes and ulnar neuropathies, Schuele [1-3]. Thus, the health care professionals and dentists must understand the background of a musician, in particular the relation between the instrument and the body, namely the type of efforts that appear while performing. Irina Foxman et al. [4] also confirmed on a survey that the time musicians are exposed to the awkward postures when playing instruments may result in playing-related musculoskeletal disorders.

Leaver et al. collected data regarding musculoskeletal pain in elite professional musicians from British symphony orchestras (243), 58\% had worked as professional musicians for more than 20 years, playing on average for $30 \mathrm{~h} /$ week [5]. Altogether $86 \%$ of the subjects reported musculoskeletal pain in the past 12 months, including $41 \%$ with disabling pain. The anatomical sites where pain was mostly referred were the neck, low back and shoulder (51-56\% in the past year) [5]. Eczema in the lip of brass players, like trumpet, tuba, french horn or trombone is also referred. Regarding the orofacial region, an injury that is well known among trumpet players due to the high pressure required to play the trumpet, is the "Satchmo's syndrome" which is the rupture of the orbicularis oris muscle. Yeo et al. dedicated his research to specific orofacial problems experienced by musicians, where orthodontic problems, soft tissue trauma, focal dystonia, denture retention, herpes labialis, dry mouth and temporomandibular joint disorders (TMD) were identified as orofacial problems of career musicians [6]. The area of TMD has been subject of several studies in professional orchestra musicians, with 408 responses to a questionnaire; TMJ pain was reported in between 15\% (lower strings) and 34\% (woodwinds) as a common problem [7]. From a sample of 41 violinists from the Murcia Regional Symphonic Orchestra, Rodríguez-Lozano et al., found that the violinist group had significantly more pain in maximum mouth opening, parafunctional habits and occurrence of temporomandibular joint sounds, which led to a conclusion that violin playing appears to be a factor associated with TMD related findings [8].

To understand the orofacial issues that are present during the performance of string and wind instrumentists, other type of parameters as e.g. accelerometers, or infrared thermography can be used for the interpretation and diagnoses of certain pathologies involving the

*Correspondence to: Miguel Pais Clemente, Dentist, PhD Student, Faculty of Dental Medicine Porto, Portugal, E-mail: miguelpaisclemente@hotmail.com

Key words: occupational healthcare, wind instrumentalists, musician's embouchure, orofacial trauma, piezo-resistive sensors

Received: March 20, 2018; Accepted: April 11, 2018; Published: April 13, 2018 
cranio-cervico-mandubular-complex (CCMC) in the case of a violin or piano player $[9,10]$.

Likewise, piezo-resistive sensors can be used to evaluate the forces that are applied by the anterior upper incisal teeth on the mouthpiece of a clarinet player. Hereafter, the authors will describe the treatment procedures of compromised central incisor of a clarinet player, that will eventually fulfill the necessary conditions for its maintenance without pain. Thus, it was produced a proper fitted appliance, the "Embouchure Stabilization Mouthpiece Appliance -ESMA", to reduce abnormal and undesired forces to the upper central incisors.

\section{Material and methods}

This study was conducted in a private dental clinic in Portugal with the collaboration of the Faculty of Engineering, University of Porto, Portugal. The patient, a single reed instrument player underwent anamneses and clinical examination, which was complemented by a panoramic X-ray. The anamneses provided data regarding the participant's personal information, medical and dental history, any existing parafunctional habits and possible temporomandibular disorders. The subject was previously informed about the procedure, the purpose and potential risks of the treatment. Since the patient was minor, the informed consent term was signed by his mother.

The clinical examination was preceded by an anamneses and history evaluation of the antecedents that were in the origin of the orofacial trauma. The vague responses of how the clarinet student fell, were inconclusive to determine the correct origin of the orofacial trauma, nevertheless it was possible to observe and confirm with the panoramic $\mathrm{X}$-ray, that this occurrence was not the first time, since the same happened before with the upper left central incisor, the 21 (Figure 1).

A special attention was also played during the stomatognathic examination to the presence of signs and symptoms of temporomandibular disorders, namely masticatory muscle pain, TMJ pain, pain during the cinematic movements of the mandible and TMJ sounds. The patient was absent of any of these conditions, nevertheless there were some signs of abrasive wear in the lower anterior incisors.

During the evaluation of the fracture present in the upper right central incisor it was possible to check the origin of tooth pain reported by the patient. In fact, with the digital pressure and observation of the respective range of movement present in the tooth, there was no report of pain. This condition was only present while playing the clarinet and during the masticatory process on the initial bite with the anterior teeth (Figure 2).

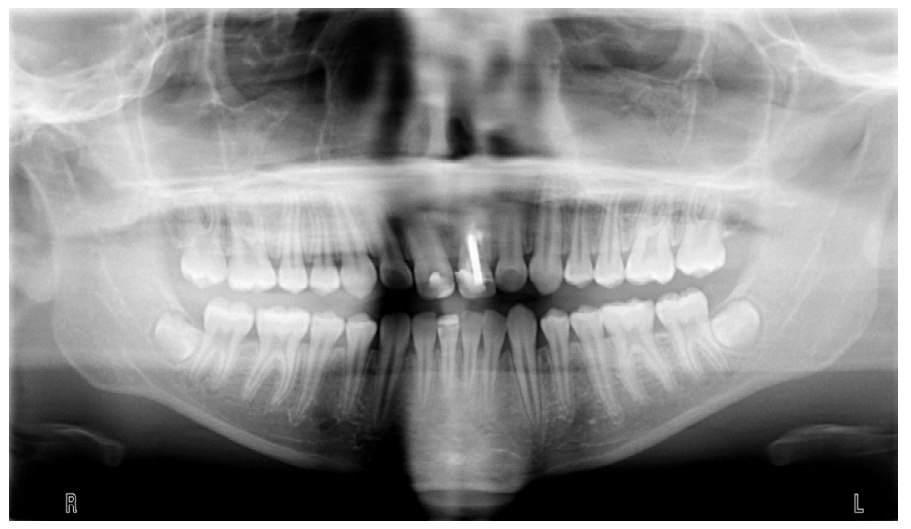

Figure 1. Initial panoramic X-ray, before dental treatment

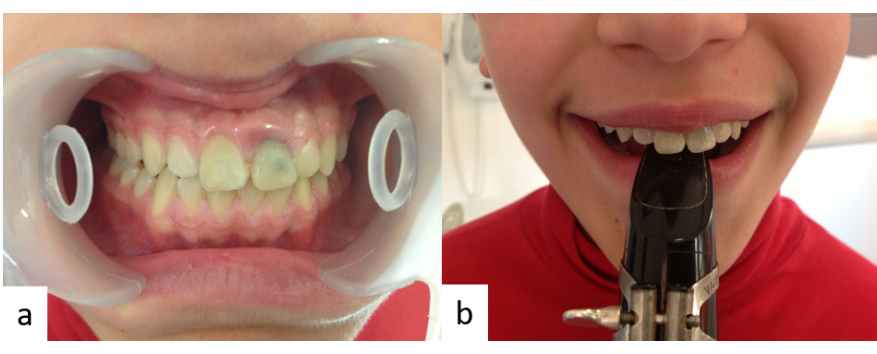

Figure 2. Orofacial trauma of the upper right central incisor (a), Clarinet player embouchure with the grip on the central incisors which was crucial for the previous stabilization of the embouchure (b)

All these situations were being avoided by the patient that showed very little inflammatory condition in the periodontal tissues surrounding the tooth. The periapical X-ray (Figure 3) showed a slight chance to carry out the root canal treatment of the tooth 11 , which was performed in one session.

The root canal was enlarged using Kerr files (Kerr, Switzerland) up to file number $80 \mathrm{k}$ and $2.5 \%$ sodium hypochlorite for irrigation and then obturated using gutta-percha and calcium hydroxide (Sealapex; Kerr, USA) with the lateral condensation technique. After this procedure, the mandatory and main condition that the authors had to deal with was the mobility and restoration of the compromised endodontically tooth. A large metallic post was placed inside the root canal with the purpose of connecting the two fragmented pieces of the root. The metallic post was cemented with glass ionomer-cement. This gave stability and stiffness to the tooth itself, showing very little mobility after the final restoration with composite resin. To prevent any kind of unwanted loading, a ORMCO wire ref ${ }^{a} 0190-264-1119$ was placed in the palatal surface of the teeth from canine to canine (Figure 4).

The following step was to obtain an accurate alginate impression of the maxillar and mandibular jaws in order to get the upper and lower casts. These were mounted in a semi-adjustable articulator after a registration bite in centric relation. The maxillar dental cast was placed in the vacuum machine (3D Motion, Erkoform), for the fabrication of the "Embouchure Stabilization Mouthpiece Appliance -ESMA". The specifications on the design of this appliance was outlined on the coverage area of the upper dental cast. A foil of $2 \mathrm{~mm}$ thick of ethylenevinyl acetate (EVA) was used, adapted and covered all the teeth of the upper jaw, which was then confined to the desirable initial design from tooth 13 to tooth 23 . This design was advocated by the authors in order to provide protection to the anterior teeth, fit the arch comfortably and have a stable retention. On the occlusal surface of this device, acrylic resin was placed in order to simulate the incisal edges of the two central incisors. Bellow this resin, a wire was placed to sustain higher forces and reduce the risk of fracture during the cyclic load applied while performing the embouchure.

Two piezo-resistive sensors (flexiforce A-201, $25 \mathrm{lb} / \mathrm{sqi}$, from Tekscan $^{\text {tix)}}$ ) were used to measure the load on the upper anterior teeth during the embouchure and consequent musical performance (Figure 5). The sensors were powered by $5 \mathrm{~V}$ DC, and connected as a voltage divider circuit to a pre-amplification before being acquired by the NI-DAQ 6008, from National Instruments. The software for data acquisition was developed in LabVIEW 2013 (from National Instruments), and implements the individual calibration characteristic of each sensor.

To be precise, the sensors were placed between the ESMA and the upper part of the mouthpiece. In fact, the objective of this appliance was 


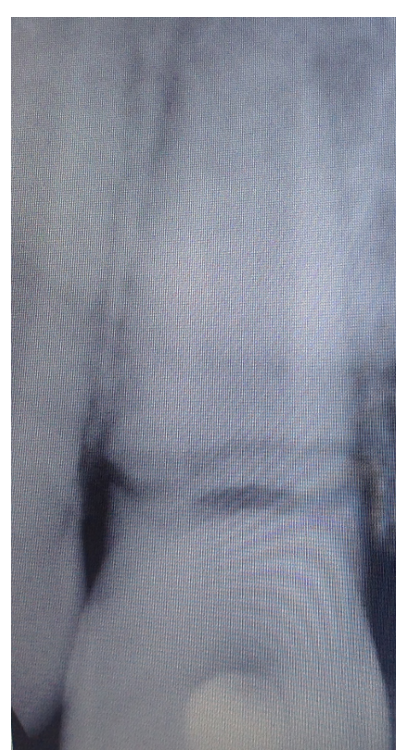

Figure 3. Periapical X-ray with tooth fracture - 11

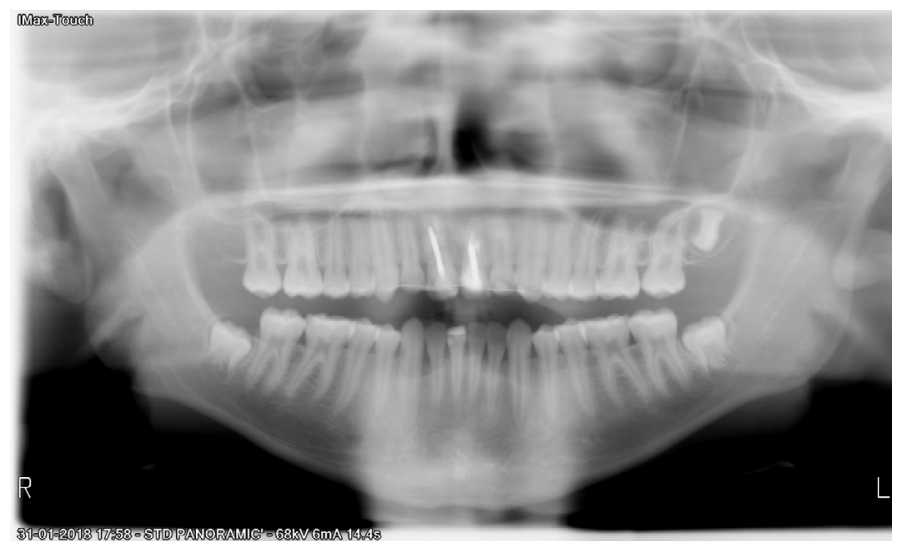

Figure 4. Panoramic X-ray with compromised tooth 11 after treatment

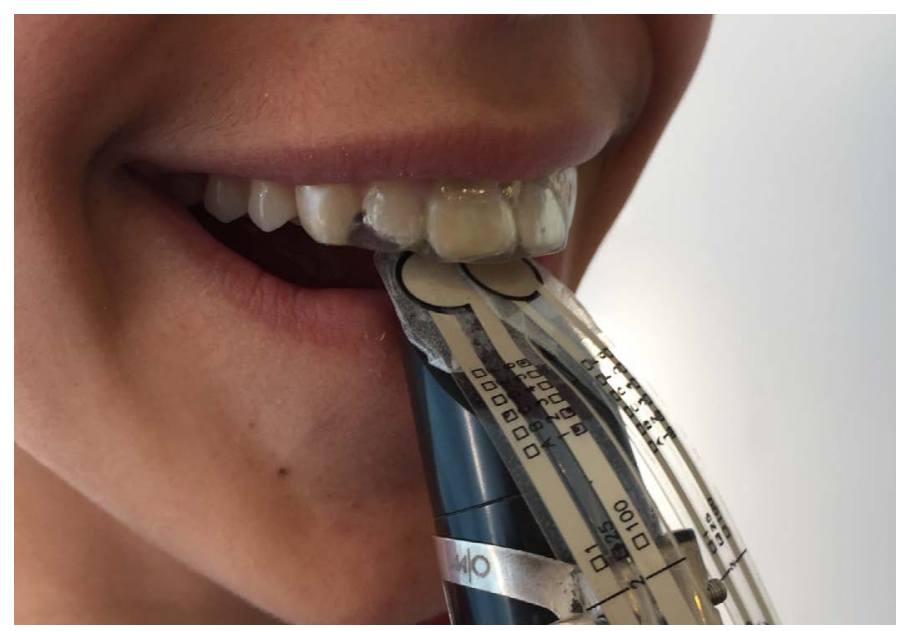

Figure 5. Evaluation of the tooth pressure with the Flexiforce sensores while wearing the homemade ESMA device

to improve the stability of the teeth and to give an additional support while playing. Since the tooth 11, by the above mentioned treatment and all the inherent conditions, have to be protected to avoid any future lesions. That was the reason why the measurements were made with the ESMA in place. This single reed instrument player was instructed to play always with this new device, as to minimize the risk of failure in the treatment plan.

At last, to contribute and achieve a neuromuscular balance of the masticatory system, an occlusal splint was fabricated. This mouthguard was made of heat-cured acrylic with a previously defined centric relation registration bite.

\section{Results}

After the root canal treatment and the respective placement of the metallic post inside the canal, there was a favorable loss at the initial mobility of the tooth. The methodology used gave a structural strength to the tooth, and allowed the musician to play without any discomfort or pain. Following the concept of improving and providing the maximum resistance to the compromised tooth, the ESMA was placed in the mouth of the musician.

This new device, ESMA, constructed for the anterior upper incisal block allowed the musician to perform and continuing playing the clarinet and obtain school success. The ESMA has a flat occlusal plane in order to simulate the "perfect" edge of two central incisors, so that the embouchure is done easily. There is a minimal increase in vertical dimension with the intention of minimizing the effort of the masticatory muscles, since there could be an increase in the muscular tonus to perform the embouchure. While playing, this device provides frictional retention to the mouthpiece and a good embouchure. Likewise, this solution makes it possible for the clarinet to play normally, nevertheless we had no idea of the amount of pressure that was being made during the musical performance. Therefore, the research findings of the force measurements were essential to confirm the good outcome of the treatment.

After the ESMA being placed in the clarinet player's mouth, a significant improvement was observed in the confidence of performing. The analysis of the measured forces was done with the average of three different registrations of a scale in a medium tone (Figure 6). The results show that the endodontic compromised tooth 11, reached a force of $0,25 \mathrm{~N}$ during musical performance, while the tooth 21 presented a maximum force of $0.5 \mathrm{~N}$. This factor is of major importance since the authors demonstrated that the use of the ESMA by the clarinet player not only protected the central incisors of the musician but allowed the force distributions on the tooth 21 being practically the double of the treated tooth 11 .

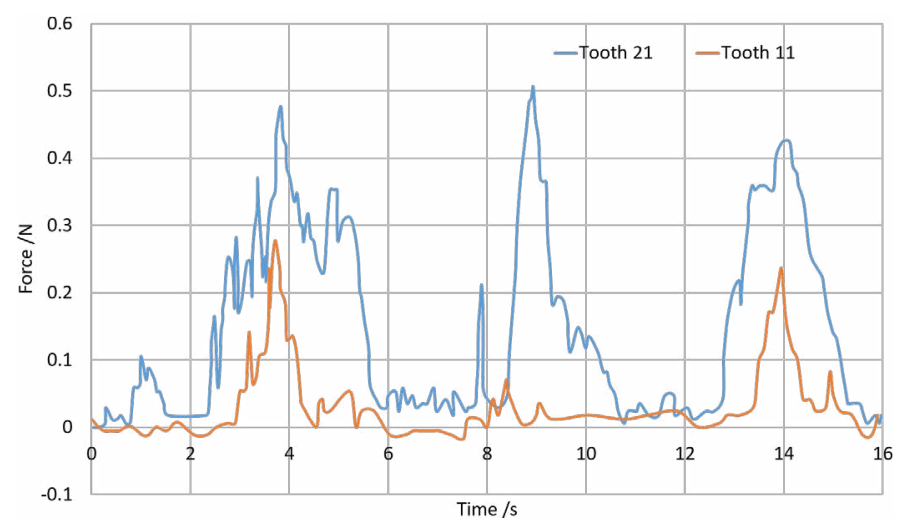

Figure 6. Force measurements during musical performance 


\section{Discussion}

The development of the ESMA and the good response obtained by the patient, gave an additional reduction in pain, providing the total absence of orofacial pain while performing. This inter-occlusal splint is a conservative and reversible treatment that was developed specially to minimize load in a compromised tooth.

Many general dentists and other dental specialists would apply the normal protocol in this kind of situation, which is the extraction of the upper right central incisor.

According to standard clinical criteria, this case could have been treated with the extraction of the tooth, and the placement of a removable prosthodontics. This removable prosthodontics should have a central screw in the palate to be adjusted every 15 days, to keep up with the normal development of the orofacial morphology. When the player reaches the adult age, it would then be possible to place an implant or a fixed bridge, as a definitive prosthodontic treatment. This is in accordance to the extension and location of the tooth fracture where the maintenance of the tooth would probably have a very low percentage rate of success.

Nevertheless, it is possible that this 14-years clarinet student would no longer be able to play clarinet with a removable prosthodontics on the upper right central incisor. Since the upper left central incisor had a previous root canal treatment and the tooth itself presented mobility. There would be many factors contributing for the instability of the embouchure, such as the adaptation of the prosthodontics, the force distribution of the mouthpiece and the proper sound quality produced.

Contrary to the substantial data for the clinical management with the previous referred therapy of tooth extraction, the authors defend that a special attention and care should be taken in order to inhibit a therapeutic that could directly or indirectly interfere in performing arts. As so, we propose the most conservative approach, in order to maintain/achieve a proper anatomical and functional solution, resulting in an "optimal embouchure". This is the embouchure that will allow the clarinet student to continue playing and have good grades.

In order to achieve good and stable results, it is fundamental to address both, dental and psychological factors. Surely that in the near future this single reed clarinet player will have to perform the extraction of the tooth 11 and undergo an oral rehabilitation, but with the work and research done it was possible minimize these interventions. This should be considered as the primary action when dealing with the embouchure of a wind instrumentalist. After three years this clarinet student continues to play, free from pain, having just a slight adjustment that was made on the appliance until the patient finishes his craniofacial growth, so he can then do a prosthodontic treatment with a dental implant. However, there are other options in the treatment of similar cases; surely that an adult would be treated in another manner. The main advantage of this procedure was the fact that the young 14-yearold boy continued playing just like his father. It was what he pointed out at the first appointment that motivated a multidisciplinary team to research and worked together to conceive and implement an effective therapeutic plan.

The engineers developed an experimental approach to study the magnitude of force applied on the clarinet mouthpiece by the upper central incisors with the ESMA placed. To our knowledge, the clinical observation of the tooth displacement presented favorable conditions of being able to sustain the stress during musical performance. The force measurement experiment allows a better comprehension of the tooth loads involved while playing. This noninvasive, highly sensitive method allowed the determination of the produced forces. The study indicates that there are light forces being applied $(0.25 \mathrm{~N})$ during short periods of time. The development of the ESMA was an important contributor factor on reducing the localized force concentration, which was dissipated through the structure of the appliance. The results provided the necessary information to determine the amount of forces that the compromised teeth were being submitted, which is vital to the clinicians and musician. This monitoring capacity is an advance in orofacial related issues of performing arts medicine making the musician feel more confident by understanding the level of force he is applying during his embouchure.

These results should be interpreted with caution because the upper right central incisor is a tooth that has a metallic post placed inside the canal with the intention of unifying a fragmented root. Usually this kind of device is placed inside the canal to give retention to the visible part of the teeth the crown.

The evidence suggests that the ESMA showed a significant outcome for the final work. Its usefulness was due to the following reasons: the clarinet player did not feel pain after the root canal treatment, there was no tooth mobility after the restorative treatment and there was no inflammatory response of the periodontal tissues. Within this general panorama, the conservative approach of this clinical case was well succeeded; the ESMA allowed and contributed for the protection of the desired structures and the single reed instrument player was able to continue playing the clarinet.

\section{Conclusions}

The findings of this study showed the importance and major role of a dentist in performing arts medicine. The constant evolution of dental techniques will enhance a better treatment for the patients in general and in this particular case of a single reed instrument player.

Nevertheless, normal standard considerations to this special case would not be probably the best solution. Being able to have knowledge and background in performing arts is essential for the final outcome of this clinical case. The chance and possibility of integrating in performing arts medicine a multidisciplinary research team with engineers and dentists working together gives confidence and raises the effectiveness in the evaluation and correction of these situations.

Clinicians should understand the biological responses of the human body, the anatomy and physiology of the involved structures, but also have a critical and objective evaluation of the best treatment plan options for the patient. The patient's needs should be considered, not only in a clinical matter, but also in the psychological aspects. In this particular case, of orofacial trauma and orofacial pain, the main goal for treatment should be the pain reduction. Health care providers should keep in mind the biopsychosocial model where the final outcome of the implemented therapies must contemplate the view of performing arts medicine. As a musical student, he is still learning and rehearsing with the single reed instrument to be a top performer, which is the natural desire of any musician. The musician's embouchure would be done with an unnatural tooth and another tooth that has an endodontic treatment with an extensive restoration. These factors will be a disadvantage in the maintenance of a correct embouchure, which should be stable, consistent and comfortable.

There will always be limitations and uncertainty towards the response of the biological tissues; however, understanding the forces that are being implemented during musical performance, should be 
considered vital. This field of bioengineering applied in performing arts, measuring and analyzing the data involving the stomatognathic function of the musician during his performance contributes for his performance and well-being. The development and application of different kinds of proper fitted appliances according to each specific need and instrument should be a reality in Performing Arts Dentistry.

\section{References}

1. Schuele S, Lederman R (2004) Long term outcome of focal dystonia in string instrumentalists. Movements Disorders 19: 43-48. [Crossref]

2. Zaza C (1998) Playing related musculoskeletal disorders in musicians: a systematic review of incidence and prevalence. Canadian Medical Association Journal 158: 10191025. [Crossref]

3. Liu S, Hayden GF (2002) Maladies in musicians. South Med J 95: 727-734. [Crossref]

4. Foxman I, Burgel B (2006) Musician health and safety. Preventing playing related musculoskeletal disorders. American Association of Occupational Health Nurses Journal 54: 309-316. [Crossref]

5. Leaver R, Harris E, Palmer K (2011) Musculoskeletal pain in elite professional musicians from British symphony orchestras. Occupational Medicine 61: 549-555. [Crossref]

6. Yeo DK, Pham TP, Baker J, Porters SA (2002) Specific Orofacial problems experienced by musicians. Aust Dent J 47: 2-11. [Crossref]

7. Steinmetz A, Zeh A, Delank KS, Peroz I (2014) Symptoms of craniomandibular dysfunction in professional orchestra musicians. Occup Med (Lond) 64: 17-22. [Crossref]

8. Rodríguez-Lozano FJ, Sáez-Yuguero MR, Bermejo-Fenoll A (2010) Prevalence of temporomandibular disorder-related findings in violinists compared with contro subjects. Oral Surg Oral Med Oral Pathol Oral Radiol Endod 109: e15-19. [Crossref]

9. Clemente M, Coimbra D, Silva A, Aguiar Branco C, Pinho JC (2015) The application of infrared thermal imaging in orchestra musicians with temporomandibular disorders. Med Probl Perform Art 30: 251-254. [Crossref]

10. Clemente M, Lourenço S, Coimbra D, Silva A, Gabriel J, et al. (2014) Three-dimensional analysis of the cranio-cervico-mandibular complex during piano performance. Med Probl Perform Art 29: 150-154. [Crossref]

11. Rodríguez-Lozano FJ, Sáez-Yuguero MR, Bermejo-Fenoll A (2010) Prevalence of temporomandibular disorder-related findings in violinists compared with contro subjects. Oral Surg Oral Med Oral Pathol Oral Radiol Endod 109: e15-19. [Crossref]

Copyright: $@ 2018$ Clemente MP. This is an open-access article distributed under the terms of the Creative Commons Attribution License, which permits unrestricted use, distribution, and reproduction in any medium, provided the original author and source are credited. 